\title{
Tensor Gravimétrico Aplicado ao Depósito de Angico, Bahia.
}

Daniel Conceição dos Santos* (IGEO/UFBA), Marcos Alberto Rodrigues Vasconcelos (IGEO/UFBA), Robson Santos da Purificação (IGEO/UFBA) e Florivaldo Oliveira Sena (SERVIGEO).

Copyright 2016, SBGf - Sociedade Brasileira de Geofísica

Este texto foi preparado para a apresentação no VII Simpósio Brasileiro de Geofísica Ouro Preto, 25 a 27 de outubro de 2016. Seu conteúdo foi revisado pelo Comitê Técnico do VII SimBGf, mas não necessariamente representa a opinião da SBGf ou de seus associados. E proibida a reprodução total ou parcial deste material para propósitos comerciais sem prévia autorização da SBGf.

\section{Resumo.}

This work makes use of the mathematical properties of the Fourier Transform to obtain the components of the Tensor gravity from data obtained in conventional gravimetric survey. Thus, an algorithm was developed in Fortran to calculate these components. The algorithm was applied to a synthetic model and its response compared to literary forecasts and subsequently applied to the gravimetric data donated by Mineração Caraíba, on the Angico area in the north of Bahia. As a product, they were generated six maps containing the tensor components, Uxx, Uxy, Uxz, uyy, Uyz and Uzz, their interpretations and correlations with the geology of the site.

\section{Introdução.}

Nos levantamentos gravimétricos, a geologia da subsuperfície é investigada com base nas variações do campo gravitacional causadas por diferenças de densidade das rochas. Os instrumentos utilizados nas medidas em campo para determinar o valor da gravidade, são os gravímetros. Os gravímetros são basicamente balanças de mola carregando uma massa constante. Variações no peso da massa causadas pelas variações na gravidade fazem variar o comprimento da mola e dão uma medida da mudança da gravidade. Os gravímetros, efetivamente, respondem apenas à componente vertical da atração gravitacional de uma massa anômala (Kearey, 2009), sendo insensíveis às bordas dos corpos, não contendo nenhuma informação da geometria da fonte (Bell et al.; 1997). Sendo a gravidade um vetor, diversos autores (Bell, 1998; Thomson et al. 2007) observaram e relataram a importância e utilidade das outras componentes da gravidade, alguns deles demonstrando que essas componentes e suas derivadas (gradientes gravimétricos) permitem determinar o campo da gravidade com maior resolução, mostrando as bordas, a forma e o centro de massa dos corpos. Estes dados adicionais, extraídos da gradiometria, encontram ampla aplicação em programas de exploração mineral e em exploração de petróleo e gás, onde é necessário o melhor entendimento das estruturas e das zonas mineralizadas. Este trabalho objetiva o emprego da técnica de cálculo dos tensores de gravidade a partir de levantamentos gravimétricos comuns, ou seja, naqueles em que foi medida apenas a componente vertical da gravidade. Assim, foi escrito um código em linguagem Fortran e aplicado a dados reais de levantamentos feitos pela Mineração Caraíba, na região do município de Jaguarari, sobre os alvos Lagoa da Mina e Cercado Velho, ambos localizados na fazenda Angicos, possibilitando um entendimento maior da geometria e mineralização desses corpos.

\section{Metodologia.}

Primeiramente foi gerado um modelo sintético, que consiste de um paralelepípedo regular com área da base medindo $2 \mathrm{~km} \times 2 \mathrm{~km}$, estando seu topo a uma profundidade de $1 \mathrm{~km}$ e sua base a uma profundidade de $2 \mathrm{~km}$, com contraste de densidade igual a $1 \mathrm{~g} / \mathrm{cm} 3$. Tal modelo foi gerado usando um pacote de algoritmos chamado Potensoft que roda dentro do software comercial MatLab. Com esse pacote, ainda, foram obtidas as componentes do tensor gradiométrico. A área do levantamento possui $10 \mathrm{~km} \times 10 \mathrm{~km}$ de dimensão, com estações distribuídas a cada $1 \mathrm{~km}$. Aos valores de gravidade obtidos com o modelo, foi aplicado o algoritmo desenvolvido e assim calculadas as componentes do tensor gravimétrico. O resultado obtido dos cálculos das componentes encontra-se demonstrado na figura 1.
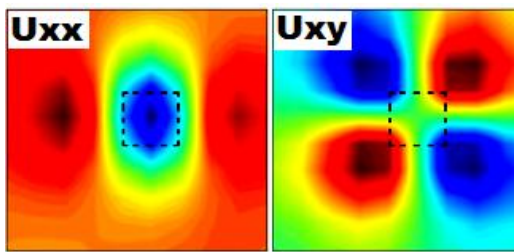

Uxz

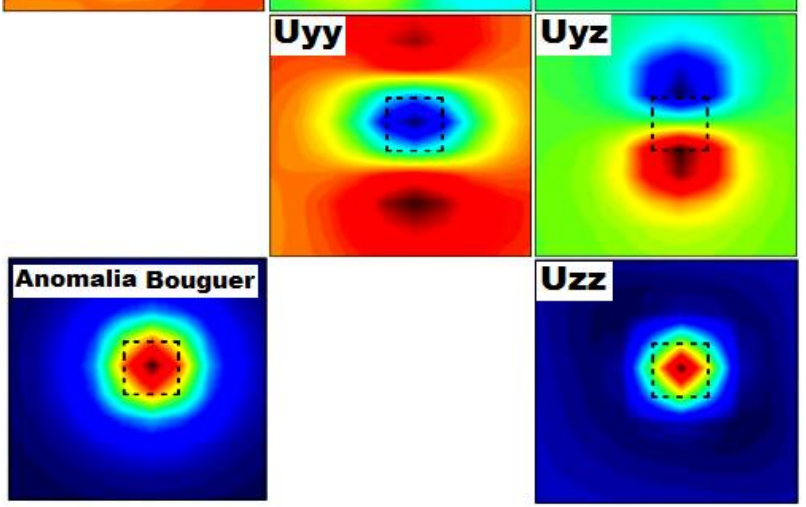

Figura 1 - Componentes obtidas com o algoritmo Fortran. 
Observou-se que os resultados estão de acordo que os previstos na literatura e com o que foi obtido com o software comercial. Assim, o passo seguinte foi aplicar o algoritmo aos dados reais de gravimetria cedidos pela Mineração Caraíbas. O algoritmo elaborado faz uso das propriedades matemáticas da Transformada de Fourier 2D.

\section{Resultados.}

Tendo demonstrado a validade do algoritmo desenvolvido ao aplicá-lo a um modelo sintético e ter obtido resultados muito satisfatórios, neste ponto do trabalho aplicamos tal algoritmo aos dados reais de anomalia Bouguer. Assim, cada componente do tensor gravimétrico foi calculada e tiveram seus respectivos mapas gerados utilizando o Oasis Montaj.
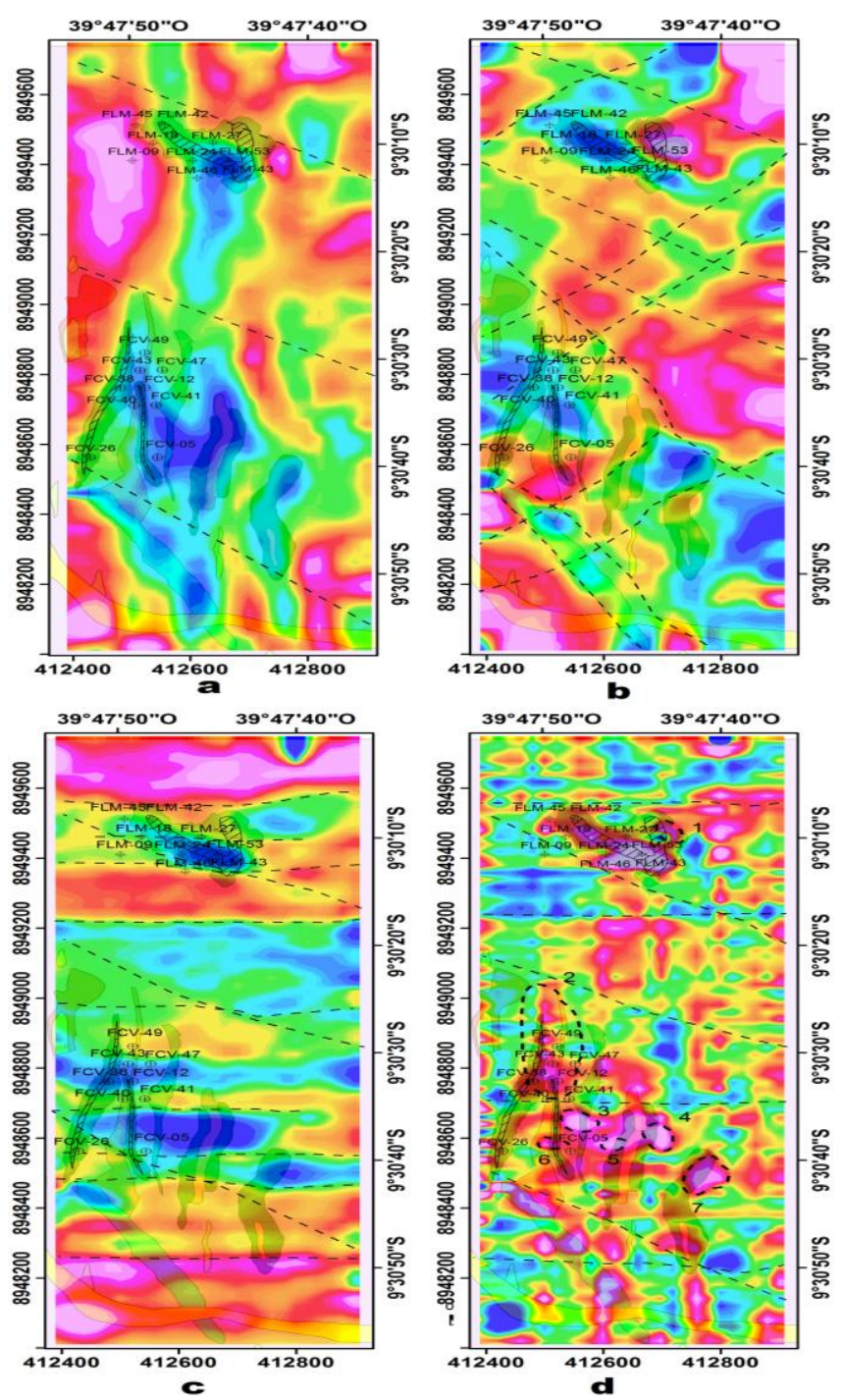

Figura 2 - (a) componente Uxx, (b) componente Uxy, (c) componente Uyy, (d) componente Uzz.Todas as componentes sobrepostas ao mapa geológico local.
A figura 2 apresenta apenas 4 das seis componentes independentes do tensor, pois foram as mais representativas para este casso particular . A figura 3 mostra a anomalia Bouguer, cedida pela Caraiba Metais, do alvo Angico que servirá para julgarmos a eficácia e contribuição do algoritmo.

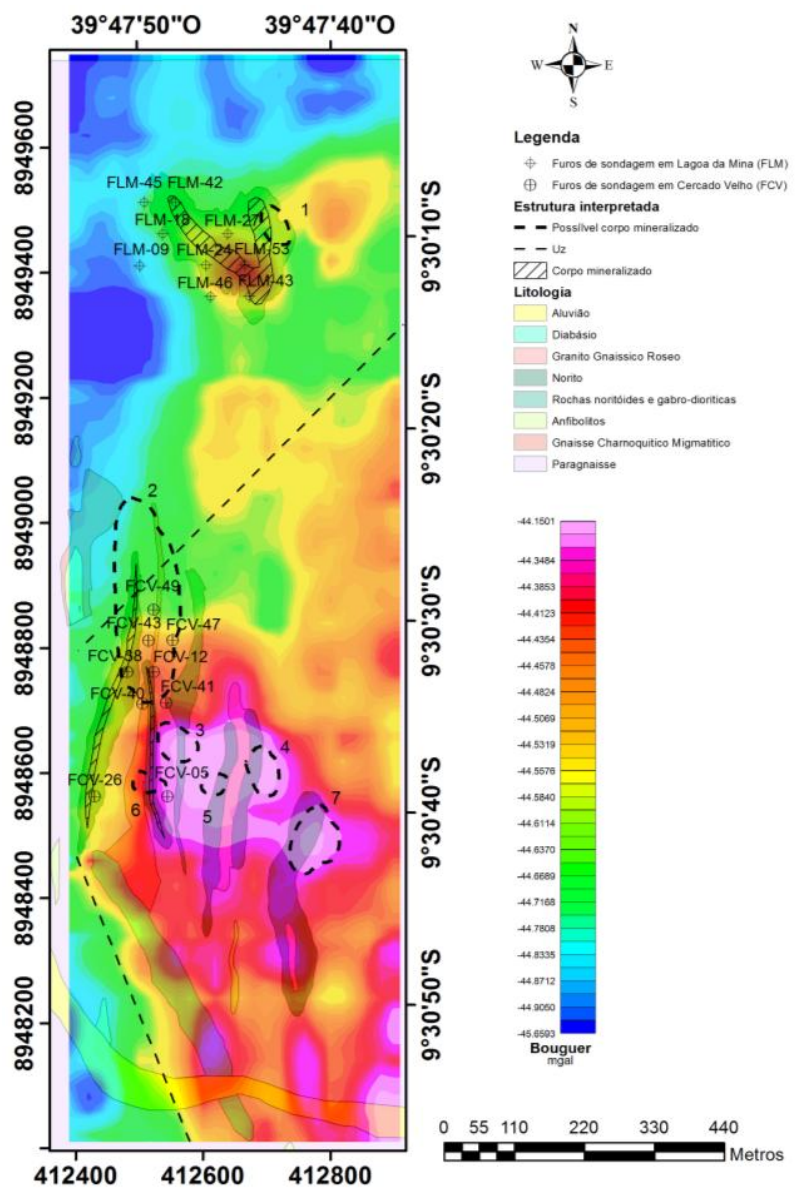

Figura 3 - Mapa da anomalia Bouguer, sobreposto ao mapa da geologia local. Fonte: Mineração Caraíba.

A partir da análise da figura 3 percebemos que a componente vertical da gravidade foi capaz de identificar o alvo Lagoa da Mina, parte ao norte, muito embora seus valores sejam relativamente baixos, mesmo estando sobre o corpo mineralizado. Deve-se notar a coerência nas direções dos furos em Lagoa da Mina (FLM). Todos esses furos possuem azimute de $90^{\circ}$, podendo interseccionar tal corpo, a menos de uma geometria muito desfavorável em subsuperfície. Na parte sul da região, em Cercado Velho, todos os corpos encontram-se representados por uma superfície suave de alto gravimétrico, ou seja, esta componente não é capaz de fornecer informações a respeito da geometria dos corpos geológicos e não se encontra correlacionada aos corpos mineralizados deste alvo. Todos os furos de Cercado Velho (FCV) possuem azimute de $270^{\circ}$, visto que a mineralização está a oeste do alvo. As regiões destacadas em 1, 2, 3, 4, 5, 6 e 7, correspondem aos corpos discretizados pela componente Uzz. Observando 
o mapa da figura 2 (a), vemos que as regiões de baixos valores de gradiometria estão associadas às bordas W-E dos corpos destes alvos. Em Lagoa da Mina, a região de baixos valores apresenta boa correlação com os limites desse corpo. O mesmo ocorre em Cercado Velho, onde é possível perceber que os corpos foram separados em duas estruturas. A estrutura a oeste do alvo é constituída por um conjunto de corpos noríticos, diabásicos, gabrodioríticos e graníticos, contendo, ainda, o corpo mineralizado. A estrutura a leste é constituída por corpo norítico e granítico. Ambas as estruturas encontram-se melhor identificadas por esta componente gradiométrica que pela componente vertical da gravidade, apenas. Observando o mapa da figura 2 (b), vemos que esta componente identificou satisfatoriamente o segmento NW-SE do corpo mineralizado em Lagoa da Mina. Já em Cercado Velho, devido à disposição espacial dos corpos, esta componente não traz informações adicionais sobre eles. Observando o mapa da figura 2 (c) percebemos que em Lagoa da Mina o baixo gradiométrico está satisfatoriamente correlacionado aos limites N-S deste corpo. Em Cercado Velho, tal fato não se repete por causa da disposição espacial destes corpos. Observando o mapa da figura 2 (d), percebemos que em Lagoa da Mina, esta componente foi capaz de identificar satisfatoriamente o corpo mineralizado. O mesmo ocorre em Cercado Velho, onde a componente foi capaz de identificar os corpos mineralizados, regiões delimitadas em 2 e 6 . As regiões delimitadas em 1, 3, 4, 5 e 7 correspondem a possíveis unidades mineralizadas nesta região.

\section{Discussão e Conclusões}

Com a aplicação do algoritmo escrito neste trabalho aos dados cedidos pela Mineração Caraíba, foi possível extrair novas informações dos dados gravimétricos. A partir da componente Uxx foi possível identificar os limites dos corpos, bem como falhas e outras estruturas na direção N-S. O mesmo é observado a partir da componente Uyy, agora na direção E-W. Da componente Uxy observamos falhas e outras estruturas nas direções NW-SE e NE-SW. Entre todas as componentes, a Uzz é a que traz resultados mais reveladores, indicando a possibilidade de novos corpos mineralizados que não são destacados pelo mapa Bouguer. Neste mapa, ainda, vemos um certo alinhamento das anomalias com as linhas de levantamento, o que pode ser gerado por desnivelamento na fonte de aquisição. Entretanto, vale lembrar, como descrito no capítulo 2, que a região possui diversos pontos de mineralização. Assim, concluo que o trabalho sugere novos pontos de mineralização. Entretanto, como todo método geofísico, deve ser correlacionado a outros métodos e à geologia, para dirimir erros e ambiguidades nas interpretações.

\section{Agradecimentos.}

Os autores agradecem: à Mineração Caraíba S/A por disponibilizar os dados do levantamento gravimétrico para a confecção deste trabalho.

\section{Referências.}

Bell R.E., Anderson R., Pratson, L., 1997. Gravity gradiometry resurfaces. The Leading Edge, v.16: 55-59.

Bell, R.E., 1998. Gravity Gradiometry. Scientific American, n.6: 74-79.

Blakely, R. J., 1996. Potential Theory in Gravity and Magnetic Applications, vol. I, Cambridge Un. Press, Australia.

Brooks, M., Kearey, P., Hill I., 2009. Geofísica de exploração, Oficina de Textos, São Paulo, 422 pp.

Delgado, I. M., Souza, J. D., 1975. Projeto Cobre Curaçá: relatório final, geologia econômica do distrito cuprífero do Rio Curaçá, Bahia, Brasil. Salvador: CPRM; Diretoria de Operações Agência Salvador, vol. 30.

Misi, A., Teixeira, J.B.G., 2012. Mapa Metalogenético Digital do Estado da Bahia e Principais Províncias Minerais. Convênio: CBPM, DNPM, UFBA -CPGG. Salvador, Bahia,

Rabelo et al., 2008. Avaliação de imagens multipolarizadas de radar (banda L) do sensor SARR99B para mapeamento geológico no Baixo Vale do Curaçá, Bahia. Revista Brasileira de Geociências. 38(4): 700-714.

Thomson, S., Fountain, D., Watts, T., 2007. Airborne Geophysics - Evolution and Revolution. The Leading Edge, 19-37. 

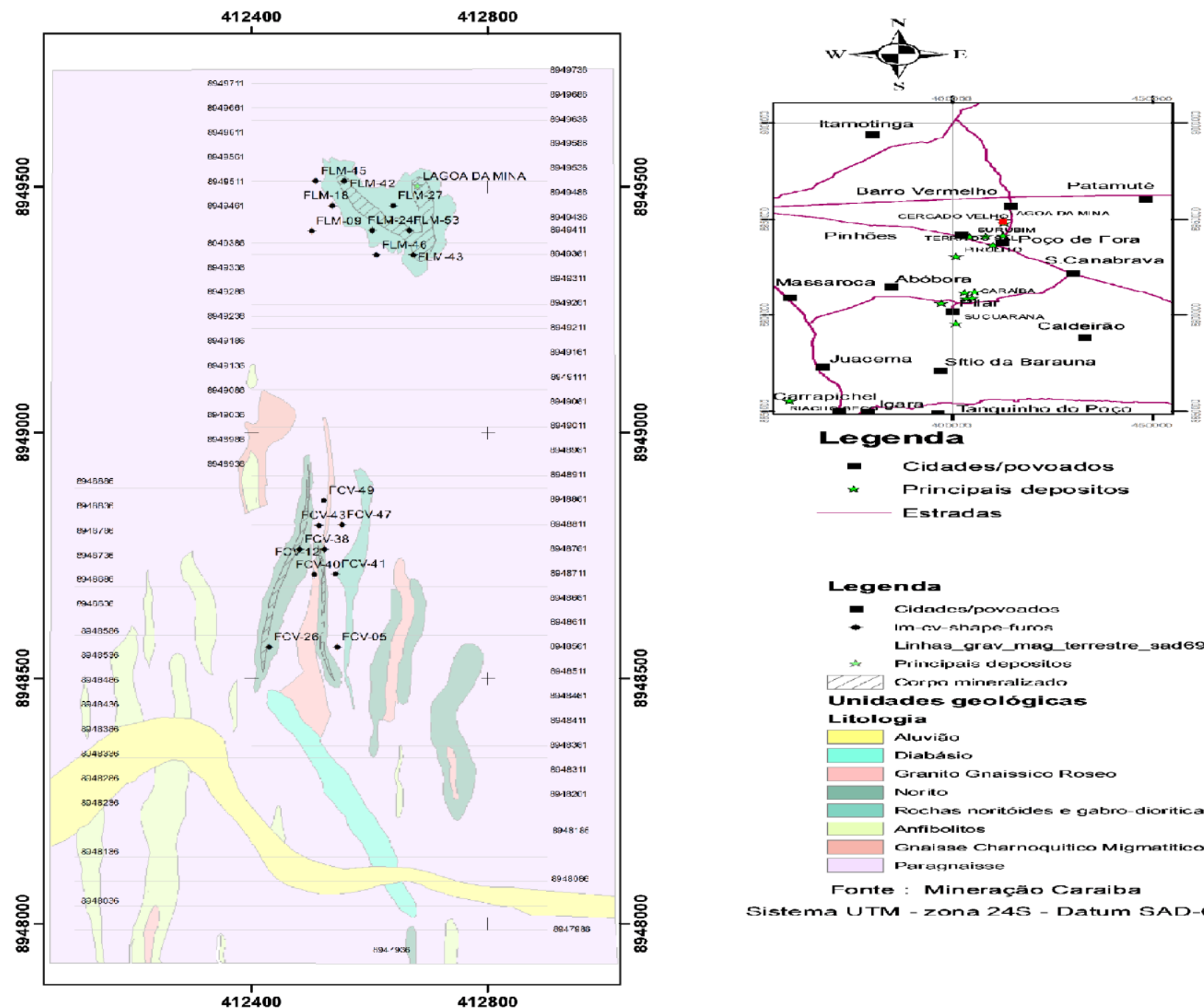

Legenda

- Cldadcospovoados

- Im-ev-shape-fums

Linhas_grav mag terrestre_sad69

* Principais depositos

PCorm mineralizado

Unidades geológicas

Litologia

Aluvião

Diabásio

Granito Gnaissico Roseo

Norito

Rochas noritóides e gabro-dioriticas

Rochas nor

Migmatitico

Paragnaisse

Fonte: Mineracão Caraiba

Sistema UTM - zona 245 - Datum SAD-G9

Figura 4 - Mapa da geologia local de fazenda Angico. 